\title{
The role of working memory in sentence processing in normal reading population. A review of related literature
}

\author{
Grammenou Anastasia \\ MSC Human Communication Sciences
}

\begin{abstract}
This essay aims to describe the factors that influence sentence processing with emphasis given on garden path sentences. The latter grammatical phenomenon has been proved more problematic in people with low working memory span. Predictions of the working memory model of Baddeley and Hich and the theory of language comprehension of Just and Carpenter were used to explain sentence processing within text context.
\end{abstract}

Index Terms-working memory, sentence processing, language processing, obstetric history

It has been more than sixty years since Hebb [1]. proposed that memory cannot be viewed as a monolithic system, instead it should be separated into long-term and short-term memory. This idea of Hebb was most influential in the following years with scientists trying to investigate a series of phenomena such as the association between short-term memory, reasoning ability and problem solving. Many models have been developed to accommodate the findings of these experiments, with the model of Baddeley and Hitch [2].being the most influential.

Baddeley and Hitch argued that many short-term memory phenomena could be better explained using the working memory concept. In their model working memory (WM) plays the role of computational arena in which both temporary storage of information and computation processing take place. The model comprises three components. The central executive which is an attention system, the articulatory loop and the visuospatial sketchpad. The former is responsible for linking the two peripheral modality dependent subsystems. The articulatory loop is the place where phonological information is stored, and the visuospatial sketchpad is responsible for manipulating visual imagery. This model failed to accommodate experimental results such as the phonological similarity effect and the word length effect. The phonological similarity effect refers to an individual's confusion observed when they are asked to recall names of phonologically similar letters. The word length effect refers to an individual's capacity limitation to recall backwards as many long words as short words. These two phenomena led Baddeley to modify the original as fixing memory model in considering the articulatory loop as comprising two devices. The phonological storage and the rehearsal process. Information can enter into phonological storage after being presented auditorily to subjects or when the subjects use their own speech production mechanism to articulate information. Rehearsals on the other hand use only articulatory coding and operate under the restrictions of speech production, that is, longer words take longer to rehearse[3].

This working memory model inspired Just and Carpenter [4]. to develop a theory of language comprehension based on individual differences of working memory. A core principle of this theory is that both information storage and processing use the same commodity, that is activation. In this view working memory capacity is synonymous to the available activation that could be used for either information storage or processing. When an individual is engaged in the process of reading information from the text is encoded in WM, either as a direct product 
of reading, or as a computational product, or as retrieved information from long-term memory. As long as these pieces of information are activated above a threshold are parts of WM and is possible for them to be manipulated by various processes. In the case when total amount of activation required for information maintenance and manipulation is less than that available, earlier stored information will be forgotten. Additionally this theory holds that syntactic, semantic and pragmatic processes involved in sentence comprehension can be executed in parallel giving partial products that will be stored in WM. However, if the processes demand an amount of activation which exceeds the available one then their attempts will be scaled back to a level that keeps activation within limits.

The previously described models of WM and language comprehension have been validated by a series of experiments that tested the relationship between information storage and sentence processing. The earlier experiments concerned subjects maintaining digits or letters while at the same time processing sentences. These experiments aimed to investigate the nature of WM capacity, determining the trade-off between storage demands of irrelevant items and the processing demands involved in sentence comprehension. Subjects were presented with a series of letters the number of which varied from one to eight. After they read the sentences they had to respond whether these were acceptable or not, holding at the same time the digits in their memory. In order to accomplish this they were instructed to articulate the sequence of digits until the verification task ended. Recall of digits took place when the verification task was completed. The results of these experiments showed that subjects took longer to respond when the digit load increased. This effect supports the idea of sentence comprehension being impaired by memory load and verifies the hypothesis that working memory holds a central role in sentence processing. [3].

The previously described experiment demonstrated that WM was responsible for storage and processing but it failed to reveal at which point performance begins to deteriorate. More precisely data from this experiment did not show that individuals with different WM capacities responded differently to the various memory loads. Thus the present experiment failed to differentiate individuals with different WM capacities.

While Baddeley was working in developing and modifying the working memory model, Daneman and Carpenter [5] developed a task that could differentiate between individuals according to their working memory capacity. This task was named Working memory Span and involved subjects reading sets of sentences while retaining the final word of the sentence in their memory. At the end of the reading process subjects had to recall the final words in correct order. Low span subjects were those who recalled at most two words. Medium Span subjects were those who recalled three or four words and high Span subjects were those who recalled five or more final words. The results of this experiment in accordance with the working memory model [2,3] and the language comprehension theory of Just and Carpenter [4] fuelled the research of individual differences in sentence processing. Working memory has been proposed to play a core role in syntactic processing due to its storage and computational properties [6] Syntactic processing transforms a linear sequence of words into hierarchic syntactic structure, a process which requires temporary storage of word representations during left to right sentence processing, until the assignment of proper thematic roles in all sentence and clause elements is completed. More demanding in terms of WM capacity appear to be the category of garden-path sentences. According to Waters and Caplan [7] garden-path sentences are "locally ambiguous syntactic structures, whose ultimate resolution is toward an unfavored syntactic representation" (p.343). 
The category of garden-path sentences comprises:

- object relative clauses such as "The reporter that the senator attacked admitted the error" [8]. In this kind of sentences the first head noun phrase is the agent of the main clause and the recipient of the action in the relative clause.

- Reduced relative clauses such as "The boat floated down the river sank. [9] The first head noun phrase is the subject of the main verb and "floated down the river is a reduced relative clause that submodifies the subject of the main verb.

- Sentence complements such as "The defendant confided to the lawyer he admired the judge was his brother." In this type of sentence the direct object of the main verb is a clause - King and Just [6] investigated the role of WM capacity in comprehending objective relative clauses versus subject relative clauses such as "The reporter that attacked the senator admitted the error," which do not generate garden-path effect.

The object and the subject relative clauses had been presented at the end of sentence sets containing none, one or two more sentences apart from the relative sentences. Subjects had to read all the sentence in a set, retaining the last word which they recalled at the end of reading. After reading and recall was competed they answered a comprehension question referring to the relative clause. Low Span readers proved to be poor at recalling words at the three sentence sets as well as at comprehending object relative clauses without any memory load. Data showed that half of the low Span readers answered the comprehension questions referred to object relative clauses at chance, that is they gave correct answers only $51 \%$ of the time, while the others gave correct answers in the same condition to $81 \%$ of the questions. This difference between low-span readers emerged because those who performed at a chance level did not spend much time associating actions with their appropriate thematic roles.

On the other hand, the performance of a quite large portion of low-span readers (50\%) in the same way as high-span readers did in the comprehension of object relative sentences task, is consistent with the core principles of the compensatory-encoding model [10]. This model focuses on the two sets of factors that can impair comprehension, subcomponent inefficiencies due to either impaired subcomponents or to the speed by which subcomponents transmit low quality of information to higher levels of language process, and low verbal working memory capacity. This model predicts that readers with inefficient access to semantic memory are able to develop metacognitive skills, such as reading back to previous part of the text or slowing down the speed of reading.

Walczyk and Taylor [11] tested the compensatory encoding mechanism of high and low span readers. They employed measurements that tagged subcomponent process such as decoding, semantic memory access, anaphor reference resolution and working memory capacity. They found that decoding skills were associated with reading back to previous parts of the text. The failure to obtain any statistically significant association between working memory capacity and reading back in text probably lies in the kind of working memory task they used. The Working memory Span was not measured with the traditional task of Daneman and Carpenter [5] but instead an adaptation of the Perfetti and Goldman task was used[12]. This task does not assess ability to retain information always located in the part of place in the sentence, but instead this assesses individuals ability to retain the whole sentence in their memory. This is because subjects were presented with two sentences, in word by word fashion, the completion of which followed presentation of a word, which occurred in one of the sentences, and subjects had to name the word immediately following.

Another plausible explanation for low span readers performing at the chance level in the King and Just [6] study is the possibility that they might have relied on pragmatic cues provided by 
sentences, in order to answer comprehension questions about object relative clauses. This hypothesis has been further pursued by the two researchers in the second experiment of their study. They tested four conditions a) when pragmatic cues were available for both man and relative object clause; b) when only the object relative clause was cued; c) when only the main clause was cued; d) neither of the clauses were cued. Low span readers were found to perform poorer when pragmatic cues were not available for the relative clauses. In contrast their comprehension of main clauses was at the same level as that of high span readers. Evaluation of these findings supports the idea of low span readers being less efficient in building sentence level interpretation based only on syntactic context. It seems that the time and effort required to make contextual information available is greater for a series of words, such as sentences, than for a single word.

The ability of high and low span readers, to benefit from sentence and lexical context when comprehending sentences, has been studied by Van Petten and his colleagues $[13,14]$ using the event-related brain potentials (ERP). A previous study by Kutas and Van Petten [15] had shown that the U400 component of ERP, which is a negative wave peaking at about $400 \mathrm{msec}$ after the onset of a visually presented word, is a sensitive index of semantic association word pairs, words lists and sentence paradigms. The reason for this is that words which are congruent with a preceding sentence context or semantically associated to a single word presented earlier, elicit smaller U400 than incongruent or unrelated words. Additionally they found that words occurring late in the sentences can benefit from preceding sentence context. eliciting again smaller U400. In their experiment Van Petten and his colleagues studied the event-related potentials elicited by high and low span readers when encountering congruent or anomalous syntactic sentences containing associated or unassociated pairs of words. The results obtained from this experiment validates the hypothesis that low span readers were less efficient in benefiting from sentence context in the absence of semantically associated words. Another result obtained from this study that merits further discussion is the inability of low span readers to benefit, to the same extent, as high and medium span readers from the presence of semantically associated words in syntactic congruent sentences. Again there seem to be two alternative explanations. The first one comes from the area of psycholinguistics and presupposes the spreading activation of semantically associated words. This phenomenon is believed to occur in sentence processing and has been studied using the cross-model priming paradigm. The latter involves priming of a target word when a semantically associated word occurs in a sentences presented earlier [16]. Experiments that investigated the processes involved in ambiguous word resolution have shown that event-related words could be primed more easily than other kinds of semantically associated words such as synonyms [17]. Van Petten and his colleagues suggested that this mechanism also operates when high and low span readers use lexical context to process anomalous sentences or sentences with ambiguous syntactic structures. If this is the case then low span readers, although able to prime semantically associated words, can't retain them for long in their working memory either due to their limited capacity or because they lack a mechanism equivalent to the rehearsals which operates for phonological information, and which would enable them to retain episodic events.

Evidence for the limited capacity hypothesis comes from studies that examined ambiguous word resolution in high and low span readers $[18,19]$. The latter group of readers were found to be impaired in holding both meanings of an ambiguous word when these differ in their frequency.

On the other hand the second hypothesis has been validated for children with severe spelling disabilities who were also manifesting impaired working memory abilities [20]. 
The second alternative explanation for low span readers inefficiency to use semantic cues derived from the preceding words in order to process syntactically ambiguous sentences, lies in the inefficient inhibitory control mechanism these individuals manifest. The inhibitory control mechanism is responsible for determining which activated representations will gain entrance into working memory, as well as for suppressing no longer relevant information $[21,22.23]$ According to this view older adults and children are less able to abandon no longer relevant information, a phenomenon that results in disruption for comprehension of gardenpath sentences [24, 25] These two age groups have been found to manifest difficulties in comprehending syntactically complex sentences such as negative sentences [26], so we might infer that this is merely due to their poor inhibitory control mechanism, despite the fact that this hypothesis has not been tested directly.

\section{THE ROLE OF WORKING MEMORY IN PROCESSING SENTENCES WITHIN TEXT}

In the first part of this essay we discussed the merits and the limitations of the capacity theory of comprehension in accounting for individual differences in processing isolated sentences or sets of irrelevant sentences [5,27]. This part of the essay deals with the role of working memory in comprehending sentences within text.

The role of working memory in text comprehension is considered to be vital as it serves as a buffer to maintain recent propositions in order to establish local and global coherence. Additionally WM holds the role of a computational arena where integration of information obtained from reading and information retrieved from long-term memory takes place [28]. The basic idea which underlies the research on text comprehension in relation to working memory capacity is that readers attempt to construct a mental model of the situation described in the text. The mental model of the text is constructed using elabolative inferences that result in more concrete representations [29]. An elaborative inference is obtained if we substitute a member from a category by the name of the category e.g. we might substitute the word "Goldfish" by the more general word "fish" [30]

The role of individual differences in $W M$ and the use of elaborative inferences in comprehending text has been studied by Whitney and his colleagues [30]. The researchers found that low span readers focus more on the initial sentence of the text in order to construct a thematic representation of it. Having accomplished this, they process further information trying to interpret it under the light of the thematic inference. Furthermore low span readers face a trade-off between holding the thematic inference and connecting successive propositions from the text.

The use of early thematic inferences (elaborations) is also responsible for another phenomenon observed in low-span readers which is manifested an inability to shift perspective when recalling a narrative text. Lee-Samons and Whitney [31] examined this phenomenon using short texts that was read by low and high span readers. The completion of reading followed a recall of the story, but from a perspective which was not given in the text. The low span readers had difficulties in shifting perspective because they did not pay attention to information which covered the alternative perspective.

Finally Budd, Whitney and Turley[32] studied the types of strategies used by low and high span readers when reading expository text. They argued that different strategies employed by individuals result in different kinds of information being recalled. Those who use thematic strategies to understand a text recall more top-down information. In contrast those who use linear strategies they recall more detailed information. The researchers varied the difficulty of the text that high and low span readers had to process by eliminating, or not, the initial 
sentence of the text. Their findings favor the idea that individual differences in WM are manifested only when the task difficulty increases. Low-span readers performed in the same way as high span readers when the initial sentence was present. On the other hand, in the initial sentence absence condition both high and low span readers had to complete a dual task, in order to construct a thematic representation at the same time as processing detailed information. This situation was able to differentiate the two ability groups as low span readers did not give as many correct response as high span readers, when questions referred to detailed information.

\section{THE CORRELATION OF OBSTETRIC HISTORY AND NORMALLY DEVELOPING CHILDREN}

1) Preterm labor is the leading cause of neonatal mortality and morbidity and associated with an increased risk of developmental problems including reading disabilities later in life $[33,34,35]$, The prematurity constitutes a premature transition from intrauterine to extrauterine life that exposes an immature central nervous system(CNS) to inadequate and painful stimuli (B) According to current avalaible literature is reported that early preterm labor ( $<32$ pregnancy week) cause to led difficulties in specific linguistic abilities which up to age 8;0 years ,even in absence of a general delay[34]. (Early insult on the fetal brain during crucial life periods of development may have long-lasting effects on congnition, reading, spelling abilities, and implications for a future child academic promotion [35]. Except the prematurity the main neonatal complications include: neonatal hospitalization, being in an incubator requiring oxygen therapy, general anesthaesia or surgery [35].

These findings confirm the importance of the perinatatal and postnatal periods in CNS development of brain regions and their contribution in general child s educations abilities

\section{DISCUSSION-CONCLUSIONS}

This essay is a negotiation of the role of working memory in sentence processing. In the first part of the essay we gave a brief description of the working memory model developed by Baddeley and Hitch [2]. as well as of the language comprehension theory proposed by Carpenter and Just [5]. This theory holds that working memory is the place where partial products of sentence processing are integrated. Furthermore, processes involved in sentence comprehension can take place in parallel as long as the capacity demands for storage of partial products and processing does not exceed the available activation levels. This theory has been employed by researchers in order to explain a series of experimental phenomena observed in the laboratory as well as in naturalistic situations. One of these phenomena, the comprehension of garden-path sentences in relation to working memory capacity, has been discussed in detail. Low span reader, were let down by garden-path effect which resulted in impoverished comprehension. Three plausible explanations were employed to account for this phenomenon. The first one refers to the limited working memory capacity which enforces low-span readers to make role assignment prior to reading the verb of the main clause, which acts as disambiguating information. The second explanation takes into account the low span reader's inefficiency in benefiting from lexical semantic cues in order to resolve syntactic ambiguity and the last explanation is a ??? of the former two also taking into account the inefficient inhibitory control mechanism which deloads working memory with information which is no longer relevant.

In the second part of the essay we discussed the role of working memory in text comprehension. Working memory is the place where information from local process is integrated with the thematic representation in order to establish both local and global 
coherence. Low span readers had been found to be impaired in constructing thematic representations and comprehending detailed information at the sametime. Furthermore, low span readers are impaired in holding bottom-up information. The later hypothesis has been validated by experiments din which low and high span readers were asked to shift perspective.

\section{References}

Hebb (1949). Organisation behaviour New York: Wiley.

Baddeley, A., \& Hitch, G. (1974). Working memory. In Bower, G., (edit). Recent advances in learning and motivating vol. VIII New York: Academic Press.

Baddeley, A. (1986). Working Memory. New York: Oxford University Press.

Just, A., Carpenter, P. (1992). A capacity theory of comprehension: Individual differences in Working Memory. Psychological Review, Vol. 99, p.422-149.

Daneman, M, \& Carpenter, P. (1980). Individual differences in working memory and reading. Journal of verbal learning and verbal behaviour, vol. 19, p.450-466.

King, J., \& Just, J., (1991). Individual differences in syntactic processing: The role of working memory Journal of memory and language vol. 30, p.580-602.

Waters, G., \& Caplan, P. (1996). Processing resource capacity and the comprehension of garden-path sentences. Memory and Cognition, vol.24, p.342-355.

Wanner, E. \&Mararsos, M. (1078) An UTN approach to comprehension. in Halle, M., Bresnan, J, Miller, G (edits), Linguistic theory and Psychological reality, 1978 Cambridge, MIT Press.

Bever, T. (1970). The cognitive basis for linguistic structures. In Hayes, J. (edit), cognition and the development of language. New York: Wiley.

Walczyk, J. (1993). Are general resource notions still viable in reading research? Journal of Educational Psychology, vol.85, p.127-135.

Walczyk, J., \& Taylor, R. (1996). How do the efficiencies of reading subcomponents relate to looking back in text? Journal of Educational Psychology vol.88, p.537-545.

Perfetti, C. A., \& Goldman, S. R. (1976). Discourse memory and reading comprehension skill. Journal of Verbal Learning and Verbal Behavior, 15(1), 33-42.

Van Petten C. (1995). Words and Sentences: Event-related brain potential measures. Psychophysiology vol.32, p.511-525.

Van Petten, G., Weckley, J, McIsaac, H., Kutas, U. (1997). Working memory capacity dissociates lexical and sentential context effect. Psychological Science, vol.8, p.238-242.

Kutas M., \& Van Petten, C. (1994). Psycholinguistics electrified: Event-related brain potential investigations. In Gernsbacher, M (edits), Handbook of Psycholinguistics. San Diego: Academic Press.

Tabossi, P., Zardon, F. (1993). Processing ambiguous words in sentences. Journal of memory and language, vol.33, p.359-379.

Williams, S., \& Colombo, L. (1995). Constraints on the range of context independent priming from ambiguous words. Psychological Review, vol.58, p.38-50.

Miyake, A., Just, U., Carpenter, P. (1994). Working memory constraints on the resolution of lexical ambiguity: Maintaining multiple interpretations in neutral contexts. Journal of Memory and Language, vol.33, p.175-202.

Hopkinds, K., Kellas, G., Paul, S.T. (1995). Scope of word meaning activation during sentence processing by young and older adults. Experimental Aging Research, vol.21, p.123-142.

Cornwall, A (1992). The relationship of phonological awareness, rapid naming and verbal memory to severe reading and spelling disability. Journal of learning disabilities, vol.25, p.532-538.

Hasher, L., Zacks, R. (1979). Automatic and effortful processes in memory. Journal of experimental psychology: general 108, p.356-388.

Stoltzfus, E., Hasher, L., Zacks, R., Olivi, U., and Goldstein, D. (1993) Investigations of inhibitions and interference in younger and older adults. Journal of Gerontology: Psychological sciences vol. 48, p.179-188. 
Stoltzfus, E. R., Hasher, L., Zacks, R. T., Ulivi, M. S., \& Goldstein, D. (1993). Investigations of inhibition and interference in younger and older adults. Journal of Gerontology, 48(4), P179-P188.

Hasher, L., Quig, L, May., C. (1997). Inhibitory control over no-longer relevant information: Adult age differences. Memory and Cognition, vol.25, p.286-295.

Hasher, Quig, May 1997;

Lorchback, I., Reimer, J., (1997). Developmental changes in the inhibition of previously relevant information. Journal of experimental psychology, vol. 54, p.317-342.

Morris, R., Gick, U., Craik, F. (1988). Processing resources and age differences in working memory. Memory and Cognition, vol.16, p.362-366.

Just, M. A., \& Carpenter, P. A. (1980). A theory of reading: From eye fixations to comprehension. Psychological review, 87(4), 329.

Trabasso, T., Suh, S (1993). Understanding text: achieving explanatory coherence trough on-line inferences and mental operations in working memory. Discourse processes, vol.16 p.3-34.

Dubois, D., \& Denis, U. (1988). Knowledge organization and instantiation of general terms in sentence comprehension. Journal of experimental psychology. Learning Memory, and Cognition, vol.14, p.604-611.

Whitney, P., Ritchie, B. \& Clark, M. (1991). Working Memory capacity and the use of Elaborative inferences in Text comprehension. Discourse Processes, vol. 14 p.133-145.

Lee-Sammons, W, \& Whitney, P. (1991). Reading perspectives and memory for text: An individual differences analysis. Journal of Experimental Psychology: Learning, Memory and Cognition, vol.17, p.1074-1081.

Budd, D., Whitney, P., \& Turley, K. J. (1995). Individual differences in working memory strategies for reading expository text. Memory \& Cognition, 23(6), 735-748.

Mathiasen R, Hansen BM, Andersen AM, Forman JL, and Greisen G.(2010). Gestalation age and basic school achievements: A national follow up study in Denmark Pediatrics. Vol.126(6):e1553-61. doi: 10.1542/peds.20090829. Epub 2010 Nov 8

Guarini A, Sansavini A, Fabbri C, Savini S, Alessandroni R, Faldella G. \& Karmiloff-Smith A. (2010) Long-term effects of preterm birth on language and literacy at eight years. J Child Lang. 2010 Sep;37(4):865-85. doi: 10.1017/S0305000909990109. Epub 2009 Aug 24

Wocadlo, C· Rieger, I (2007). Phonology, Rapid Naming And Academic Achievement In Very Preterm Children At Eight Years Of Age. Early Humman Development, vol.83(6), 367-77. 